\title{
Wie Führungskräfte Informationen gewinnen und Meinungen bilden
}

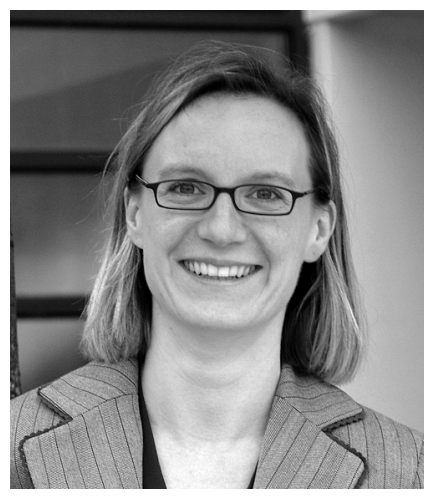

VON MONIKA SAGMEISTER Dr. Monika Sagmeister ist Abteilungsleiterin für die diözesanen Kitas im Erzbischöflichen Ordinariat München und Freising. Das Erzbistum ist Träger von 34 Kindertagesstätten mit über 400 Mitarbeiterinnen und Mitarbeitern. Sie hat Sozialpädagogik und Sozialmanagement studiert und an der Universität Regensburg promoviert.

Dioezesane-Kitas@eomuc.de

\author{
Formelle und informelle Netzwerke zwischen \\ Leitungskräften können ein bedeutsamer \\ Erfolgsfaktor für den Umgang mit Veränderungen \\ in sozialen Organisationen sein.
}

Die Sozialwirtschaft sieht sich derzeit vielfältigen Veränderungen ausgesetzt. Beispielhaft lassen sich einige Herausforderungen nennen, wie etwa die im Grundgesetz verankerte Schuldenbremse, die angespannte finanzielle Situation der Kommunen und der zunehmende Wettbewerb.

Dadurch sehen sich viele Geschäftsführerinnen und Geschäftsführer vermehrt mit Schwierigkeiten konfrontiert, ihre sozialen Einrichtungen und Angebote ausreichend mit finanziellen Mitteln auszustatten. Doch auch der Fachkräftemangel insbesondere im Bereich der Pflege und der Kindertagesbetreuung beschäftigen die Sozialwirtschaft. So lassen sich im Kindertagesbereich nur unter Einhaltung der vorgeschriebenen Fachkraftquote Angebote refinanzieren.

Gleichzeitig bauen insbesondere Einrichtungen der Freien Wohlfahrtspflege und kleinere Vereine auf bürgerliches Engagement. Dieses Engagement hat sich aber in den letzten Jahren verändert. Die Engagierten binden sich nicht mehr über viele Jahrzehnte an eine Einrichtung (Priller, 2010) und teilweise wird es schwierig, geeignete Personen etwa für einen Vorstandsvorsitz einer sozialen Einrichtung zu finden.

Zu guter Letzt verändert sich aber auch die Klientel. Die Zunahme von befristeten Arbeitsverträgen, Leih- und Teilzeitarbeit und der Ausbau des Niedriglohnsektors erschweren oftmals einen Aufstieg in gesicherte Einkommensverhältnisse (KlausSchelletter \& Kolf, 2013). Die Folge: Menschen mit multiplen Problemlagen suchen Unterstützung in den sozialen Sicherungssystemen und somit auch in der Sozialwirtschaft (Schneider, 2011).

\section{Vernetzung als Quelle neuer Informationen}

Die Geschäftsführung einer sozialen Einrichtung trägt die Verantwortung, ihre Einrichtungen auf diese Veränderungen vorzubereiten und mit ihnen umzugehen. Für sie stellt sich die Frage, wie eine Führungskraft diese Veränderungen bewältigen kann.

Eine derzeit viel diskutierte Fragestellung und nahezu ein Allheilmittel im Umgang mit den oben beschriebenen Herausforderungen scheint der Begriff der »Vernetzung « zu sein. Vernetzung kann jedoch sehr vielfältige Schwerpunkte haben. Es können Angebote vernetzt werden, um Kosten zu reduzieren. Oder es werden gemeinsame Aktionsbündnisse gestartet, um Einfluss auf politische Entscheidungsträger zu nehmen.

Häufig ist der Beginn einer Vernetzung der Informationsaustausch von Personen. Bei der Vernetzungsdebatte wurde bisher meist der Aspekt außer Acht gelassen, unter welchen Bedingungen ein erfolgreicher Informationsaustausch zustande kommt. Wer sind also die entscheidenden Personen, mit denen man sich vernetzen sollte? Was zeichnet Netzwerke mit diesen Personen aus? Und welche Attribute bringen Personen mit sich, von denen man relevante, sprich wirklich neue Informationen erhält und somit an bisher nicht zugängliches Wissen gelangt.

Um diese Fragen zu klären, wurde im Sommer 2012 von der Autorin eine Studie in Form einer egozentrierten Netzwerkanalyse (Trappmann, Hummell \& Sodeur, 2011) mit 15 Geschäfts- 
führerinnen und Geschäftsführern der Freien Wohlfahrtspflege durchgeführt. Die Befragten sind auf Kreisebene tätig und vertreten sowohl ländliche als auch städtische Regionen. Sie haben mindestens 40 Mitarbeitende. Bei dieser Studie wurden die Geschäftsführerinnen und Geschäftsführer gebeten, maximal fünf Kontaktpersonen zu nennen. Die Kontaktpersonen sollen Anregungen geben, wie die finanzielle Absicherung der vorhandenen Dienste und Einrichtungen zu gewährleisten sei.

Die 15 Führungskräfte gaben Auskunft über 73 für sie wichtige Kontaktpersonen und beschrieben neben netzwerktechnischen Fragestellungen auch die Dimensionen sozialer Unterstützung, die ihnen durch die Netzwerkkontakte zu Teil werden. Es wurden Fragen erfasst, wer wen wie oft trifft, welche Qualifikation die Kontaktpersonen mitbringt, welchen Beruf sie ausübt usw. Es wurde aber auch erfasst, inwieweit die Netzwerkkontakte zum Informationsaustausch beitragen und somit bei der Bewältigung der anstehenden Herausforderungen hilfreich sind.

\section{Die beliebtesten Netzwerkpartner}

Die wichtigsten Ergebnisse der Netzwerkanalyse: Nahezu 70 Prozent aller Kontakte werden innerhalb des eigenen Verbandes gepflegt. Etwas über die Hälfte davon $(38,4 \%)$ entfallen auf Mitarbeiterinnen und Mitarbeiter der eigenen Organisation. Diese sind entweder in der Bereichsleitung oder in der Buchhaltung tätig oder mit Verwaltungsaufgaben betraut. Weitere wichtige Netzwerkpartner sind Vorgesetzte, Ansprechpartner im Spitzenverband und

\section{Merkmale starker und schwacher Netzwerk-Beziehungen}

Der amerikanische Soziologe Mark S. Granovetter (geb. 1943) hat sich vor allem mit Netzwerkanalysen einen Namen gemacht. Seine Studien arbeiteten die Merkmale von starken (»strong ties») und von schwachen (»weak ties») Beziehungen in sozialen Netzwerken heraus:

- Strong tie: In einer starken Beziehung investieren die Akteure viel Zeit im Hinblick auf Intimität und Emotionalität. Die Erwartungen beruhen auf Gegenseitigkeit. Vorteile sind:

- unmittelbarer Schutz

- Vertrauensbildung im engen Kreis

- Zeitersparnis bei der Informationsbeschaffung

- schnelles Zusammenarbeiten

- Ressourcen lassen sich auf mehrere Situationen anwenden

Quelle: Granovetter, M. S. (1973). The strength of weak ties. American journal of sociology, 78 (1), 1360-1380.

sonen im Netzwerk haben Erfahrung im sozialen Bereich (95,9\%). Geschätzt wird aber nicht nur die generelle Erfahrung in der Sozialwirtschaft, sondern häufig wird eine ganz bestimmte Kompetenz im Umgang mit den Veränderungen nachgefragt. Im Vordergrund stehen Kenntnisse in Finanzierung und Erfahrung im Umgang mit Veränderungen $(56,1 \%)$.

\section{Was Informationsnetzwerke auszeichnet}

Es lässt sich feststellen, dass Geschäftsführerinnen und Geschäftsführer sehr unterschiedliche Vorstellungen darüber haben, welche Kontakte für sie bei der Bewältigung aktueller Herausforderungen von Nutzen sind. Die Struktur der 15 Netzwerke unterscheidet sich trotz der vorgegebenen Beschränkung auf die

\section{"Informelle Kontakte sind der beliebteste Weg zur Informationsgewinnung"}

geschäftsführende Kollegen des eigenen Verbandes. Die geringste Bedeutung haben private Verbindungen.

Der beliebteste Weg, um an Informationen zu kommen, sind informelle Kontakte. Formellen Treffen wird nicht die gleiche Bedeutung eingeräumt. Der wichtigste Kommunikationsweg ist das Gespräch bei einem persönlichen Treffen oder am Telefon. Nahezu alle Per- fünf wichtigsten Ratgeber stark hinsichtlich der Anzahl der Kontakte der Netzwerkpartner untereinander oder der gegenseitigen Erreichbarkeit.

Der Informationsaustausch zwischen den Leitungskräften und dem Netzwerk beruht in der Regel auf Gegenseitigkeit. Die Nachfrage und Hilfestellung ist in elf von 15 Netzwerken ausgewogen. Dies ist aber auch der Tat-
- Weak tie: Schwache Beziehungen sind lockere Verbünde mit geringer Intensität. Vorteile sind:

- Informationen aus der Außenwelt

- Erhöhung der eigenen Sichtbarkeit

- mehr alternative Denk- und Verhaltensweisen, Sichtweisen und Meinungen

- zusätzliche und neue Informationen

- frühzeitige Wahrnehmung neuer und zusätzlicher Informationen

sache geschuldet, dass die Mehrheit der Netzwerke durch persönliche Kontakte entstanden sind, die bereits seit längerem bestehen sich über Jahre hinweg entwickelt haben.

Netzwerke können je nach Zielsetzung durchaus strategisch eingesetzt werden. Durch den Austausch von Informationen mit einflussreichen, kompetenten Partnern entstehen neue Handlungsmöglichkeiten für das Individuum. Führungskräfte können in Netzwerken vielfach Informationen erhalten, $\mathrm{zu}$ denen sie sonst keinen Zugang haben. Gleichzeitig können Netzwerkkontakte eine Empfehlung für eine Person sein, da die Art des Umgangs und die im Netzwerk befindlichen Personen Rückschlüsse auf das eigene Sozialkapital zulassen (Henning, 2010).

Ob die durch die Netzwerkverbindungen gewonnene Information aber tatsächlich neu ist, hängt mit der Intensität des Kontaktes zwischen dem Individuum und seinem Netzwerkpartner zusammen. Unterschieden wird hierbei in starke Beziehungen, sogenannte »strong ties « und schwache Beziehungen, den "weak ties" (siehe Kasten). Nach Granovetter (1973) hängt die Unterscheidung zwischen "strong ties « und »weak ties « davon ab, wieviel Zeit im Hinblick auf Intimität und Emotionalität miteinander verbracht wird und welche Hilfeleistungen vorhanden sind.

Wenn Personen in mehr als nur einem Kontext miteinander verbunden sind, ihre Arbeitskollegin oder Arbeitskollegen also auch noch in der Nähe 


\section{Wenn Netzwerke zur Gefahr werden: Gruppendenken}

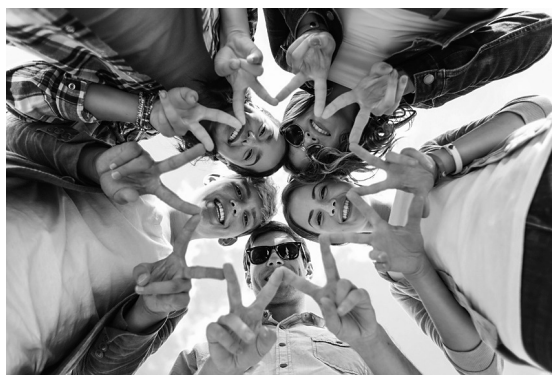

Gruppendenken ist ein Prozess, bei dem eine Gruppe von an sich kompetenten Personen schlechtere oder realitätsfernere Entscheidungen als möglich trifft, weil jede beteiligte Person ihre eigene Meinung an die erwartete Gruppenmeinung anpasst. Daraus können Situationen entstehen, bei denen die Gruppe Handlungen oder Kompromissen zustimmt, die jedes einzelne Gruppenmitglied unter

wohnt oder Freizeitinteressen geteilt werden und die Personen sich mindestens einmal die Woche treffen, sollte es sich um einen Menschen handeln, der ähnliche Interessen verfolgt. Es wird davon ausgegangen, dass diese Menschen über homogene Einstellungen verfügen und füreinander berechenbare Werte und Normen an den Tag legen.

Der Hauptvorteil solch einer multiplexen Beziehung besteht darin, dass sich Ressourcen, die sich in einem bestimmten Kontext entwickelt haben, auf andere Situationen übertragen lassen (Fliaster, 2007; Scheidegger, 2010). Sie sparen Zeit bei der Informationsbeschaffung und minimieren das Risiko, sich auszugrenzen. Dadurch entwickelt sich Zuverlässigkeit und ein Gefühl der Verpflichtung gegenüber den Netzwerkmitgliedern.

Allerdings schotten sich Personen mit engen Kontakten auch nach außen ab. Querdenker sind nicht willkommen. Dadurch werden Informationen und Einflüsse außerhalb der eigenen Werte und Normen nicht an das Netzwerk herangetragen. Wenn Sie sich also ausschließlich mit Personen aus dem eigenen Haus vernetzen, kann diese Gefahr durchaus bestehen. Wenn Sie an wirklich neue Ideen, neue Informationen und neues Wissen gelangen wollen, müssen andere Netzwerkkontakte gesucht werden. Von Vorteil sind hier »weak ties«. Je mehr sich das eigene Profil von dem der Netzwerkpartner unterscheidet und normalen Umständen ablehnen würde. Faktoren, die das Auftreten von Gruppendenken wahrscheinlich machen, sind eine hohe Gruppenkohäsion (Nahverhältnis, Ähnlichkeit, Zusammenhalt), strukturelle Mängel im Gruppenaufbau, Abschottung nach Außen, ein sehr starker, dominanter Meinungsführer im Innern, fehlende Objektivität seitens der Führungskraft, mangelhafte oder sogar fehlende Normen und Prozesse, um systematisch Handlungsalternativen abzuwägen, Bestehen einer (im Gruppenempfinden) bedrohlichen Situation, die starken Stress und viel Emotionalität auslöst.

Quelle: http://de.wikipedia.org/wiki/ Gruppendenken

je geringer die Häufigkeit und Intensität ihrer Begegnung ist, desto höher ist die Wahrscheinlichkeit, im Austausch zusätzliche und vor allem neue Informationen zu erhalten.

\section{Wissensaustausch in Geschäftsführernetzwerken}

In den in der Studie identifizierten Netzwerken ist der Wissensaustausch überwiegend nicht organisiert und findet nur dort regelmäßig statt, wo sich alle Netzwerkmitglieder kennen. Netzwerke, in denen sich alle Mitglieder kennen, bestehen allerdings überwiegend aus Mitgliedern der eigenen Institution. Hier handelt es sich meist um enge Verbindungen. Beachtenswert ist in diesem Fall die Gefahr, redundantes Wissen zu erhalten und die eigene Meinung aufgrund der geteilten Werte und Normen lediglich bestätigt zu bekommen.

Ein erfolgreicher Wissensaustausch in einem Netzwerk hängt nicht mit strukturanalytischen Gesichtspunkten des Netzwerkes zusammen. Die Häufigkeit des Kontaktes spielt somit für den gewinnbringenden Wissensaustausch keine Rolle. Von den Teilnehmenden an der Studie wird bestätigt, dass durch den Austausch im Netzwerk die Möglichkeit besteht, die eigenen Fähigkeiten als GeschäftsführerIn auszubauen. 75,3 Prozent aller Kontaktpersonen wird diese Option zugeschrieben.
Die beste Basis dazu bietet die Vernetzung mit geschäftsführenden Kolleginnen und Kollegen. Bevorzugt werden dabei Personen aufgesucht, die Motivation, Anerkennung, Wertschätzung und Orientierung bieten. Des Weiteren lassen sich die eigenen Fähigkeiten bei Personen ausbauen, mit denen die Geschäftsführung neue Geschäftsbereiche erschließen oder Informationen über Veränderungen in der Sozialwirtschaft austauschen kann.

\section{Merkmale gewinnbringender Informationsnetzwerke}

Netzwerke bieten also die Möglichkeit, die eigenen Fähigkeiten als Geschäftsführerin oder Geschäftsführer auszubauen. Die beste Bedingung dazu bietet ein vertrauensvoller Umgang mit geschäftsführenden Kollegen. Dabei spielt es keine Rolle, wie intensiv der Kontakt ist. Vielmehr ist es von Bedeutung, viele vertrauensvolle, lockere Verbünde zu organisieren, die dazu führen, dass man an Informationen gelangt, die sonst außerhalb der eigenen Reichweite liegen.

\section{Literatur}

Fliaster, A. (2007). Innovationen in Netzwerken. Wie Humankapital und Sozialkapital zu kreativen Ideen führen. München: Rainer Hampp Verlag.

Granovetter, M. S. (1973). The strength of

weak ties. American journal of sociology, 78 (1), 1360-1380.

Klaus-Schelletter, S. \& Kolf, I. (2013). Analyse des Vierten Armuts- und Reichtumsberichts der Bundesregierung. Sozialmagazin, 38 (3-4), 6-15 Priller, E. (2010). Vom Ehrenamt zum zivilgesellschaftlichen Engagement. Zeitschrift für Erziehungswissenschaft, 13 (2), 195-213.

Trappmann, M., Hummell, H. J. \& Sodeur, W. (2011). Strukturanalyse sozialer Netzwerke. Konzepte, Modelle, Methoden (2. überarb. Auflage). Wiesbaden: VS Verlag für Sozialwissenschaften.

Sagmeister, M. (erscheint 2014). Netzwerke in der Freien Wohlfahrtspflege. Umgang mit Veränderungen durch Vernetzung der Führungskräfte. Hamburg: Verlag Dr. Kova. Scheidegger, N. (2010). Der Einfluss von Netzwerkstrukturen auf den Karriereerfolg im Management: Bridging oder Bonding Ties? Netzwerkanalyse in einem Schweizer Dienstleistungsunternehmen. Rainer Hampp Verlag. 